\title{
Condylar Changes in Patients after Orthodontic Treatment - A Retrospective Panoramic Study from Chennai, India
}

\author{
Deepigaa Manivasagam¹, Arvind Muthukrishnan² \\ 1,2 Department of Oral Medicine and Radiology, Saveetha Institute of \\ Medical and Technical Sciences, Kuthambakkam, Tamil Nadu, India.
}

\section{ABSTRACT}

\section{BACKGROUND}

Idiopathic condylar resorption (ICR) is a progressive, extensive loss of condylar resorption with or without temporomandibular joint (TMJ) symptoms. Orthodontic treatment causes abnormal joint loading, thus sustained physical stress to the articular surface of TMJ when exceeds adaptive capacity leads to dysfunctional remodelling of the mandible. Condylar changes were not assessed both during pre and post orthodontic treatment. The purpose of this study was to assess the condylar changes following orthodontic treatment using orthopantomogram (OPG).

\section{METHODS}

This retrospective study was conducted in a university dental hospital setting covering patients visiting for orthodontic treatment from October 2020 to February 2021. Analysis of pre-operative and post-operative OPG's of patients who have undergone orthodontic treatment was done. Morphologic changes of condyle were recorded using idiopathic condylar resorption subgroups classification. The statistical software used for analysis was IBM Statistical Package for Social Sciences (SPSS 23) and a paired t test was used to assess the pre-operative and postoperative condylar changes.

\section{RESULTS}

50 condyles of 25 patients (right and left side) ranging from 11 - 40 years of age were evaluated pre and post orthodontic treatment. Female predilection (60\%) with phenotype 1 (44\% - right; $44 \%$ - left) being more common and left condyle most commonly affected. The mean and S.D. of the right side were - 680 and 0.748 respectively (P value 0.000 ) and left side values were - 800 and 1.118 respectively which was also statistically significant (P value 0.002$)$.

\section{CONCLUSIONS}

ICR is the result of many predisposing and contributory factors and an accurate and timely diagnosis of this pathology is essential in dental practice. The available literature confirms the presence of condylar changes after orthodontic therapy, however more evidence-based high-quality clinical trials with detailed design and long-term follow-up periods need to be conducted yet in order to gain more insight and knowledge on the onset and progression of this pathology.

\section{KEY WORDS}

Idiopathic Condylar Resorption (ICR), Temporomandibular Joint (TMJ), Orthodontic Treatment
Corresponding Author: Dr. Arvind Muthukrishnan, Saveetha Institute of Medical and Technical Sciences, Kuthambakkam, Tamil Nadu, India.

E-mail: arvindm@saveetha.com

DOI: $10.14260 /$ jemds/2021/736

How to Cite This Article: Manivasagam D, Muthukrishnan A. Condylar changes in patients after orthodontic treatment - a retrospective panoramic study from Chennai, India. J Evolution Med Dent Sci 2021;10(42):36283632, DOI: $10.14260 /$ jemds/2021/736

Submission 03-06-2021,

Peer Review 25-07-2021

Acceptance 03-08-2021,

Published 18-10-2021.

Copyright (C) 2021 Deepigaa Manivasagam et al. This is an open access article distributed under Creative Commons Attribution License [Attribution 4.0 International (CC BY 4.0)] 


\section{BACKGROUND}

Idiopathic condylar resorption is the extensive and irreversible resorption of the condyle and loss of ramus height with or without temporomandibular joint symptoms. ${ }^{1}$ It is commonly known as progressive condylar resorption ${ }^{2}$ and also as cheerleader syndrome as it commonly affects younger girls participating in sports activities where a minor trauma can initiate or exacerbate the condition. ${ }^{3}$ Persistent physical stress or abnormal loading to the articular structures of the temporomandibular joint, causes an increase in the normal adaptive capacity which leads to joint remodelling. This in turn results in a series of morphological abnormalities, such as decrease in the volume of the condyle and height of the ramus, increase in the retrusion of the mandible, or decrease in the mandibular growth.4-6 The clinical presentations of ICR usually includes rapid loss of condylar volume and decrease in ramus height. The progress in condylar resorption in the process, causes retrusion of the mandible and an increase in the mandibular plane angle and anterior face height, which results in class II malocclusion or an anterior open bite. ${ }^{7}$ The most common clinical symptom of patients with ICR include alteration of masticatory function followed by other symptoms such as facial deformity (e.g., skeletal class II malocclusion and mandibular deviation), TMJ symptoms like clicking, pain, and limited mouth opening may also exist. ${ }^{8} 25 \%$ of patients do not present any symptoms in the early stage of ICR making an early diagnosis and intervention difficult. Generally accepted treatment guidelines for this disease are also not available; as the classification and aetiology of ICR are not fully understood. ${ }^{9}$

Condylar resorption, on the basis of clinical manifestations has been classified by Hoppenreijs into 2 types - the open bite type and the deep overbite type. The risk of condylar resorption was found to be increased with the severity of open bite.10 With advancement in radiological techniques, ICR is classified on the basis of differences in condylar morphology. ICR was initially evaluated based on the contour and axis of the condyle using 2-dimensional (2-D) images. ${ }^{11}$ Different condylar types were also identified in association with the similarity of the condylar surface using 3-dimensional (3-D) mesh models. ${ }^{12}$ Orthodontic treatment and/or orthognathic surgery serve as one among the major criteria for patients affected by ICR, thereby resulting in occlusal instability, improper maxillomandibular skeletal relationships, dysfunction of the TMJ and pain. ${ }^{13-15}$

Thus, the aim of the study was to assess condylar changes in patients who underwent orthodontic treatment.

\section{METHODS}

This retrospective study was conducted in a university dental hospital setting covering patients visiting for orthodontic treatment. The study was carried out by 2 members, the primary researcher and a department faculty from October 2020 to February 2021. Sample size calculation was done using $G$ power analysis software and calculated to the power of 80. Analysis of pre-operative and post-operative OPG's of patients who have undergone orthodontic treatment was done. We included patients who have completed the orthodontic treatment and their entire records were accessible. Patients with a previous history of rheumatologic disease, systemic joint diseases, developmental deformities, previous TMJ treatment, or a history of craniofacial surgery or trauma were excluded in our study. The case sheets were cross verified based on clinical diagnosis, radiographic findings and treatment done. The data was verified by both researchers. The internal validity was maintained as set diagnostic criteria was followed and this methodology can be replicated at other centres also, maintaining the external validity also.

According to the contour of condyle, ICR was classified into 3 subgroups(Figure 1) In the ICR I subgroup, the resorption did not reach the maximum transverse diameter of the condyle thus giving a S-shaped appearance while in ICR II subgroup, the resorption has reached the maximum transverse diameter thus an angular shape and in ICR III subgroup, the resorption is more severe causing a loss of normal condylar morphology thus representing a straight contour. ${ }^{16}$

\section{Statistical Analysis}

The statistical software used for analysis was IBM SPSS 23. A paired $t$ test was used to assess the pre-operative and postoperative condylar changes.

\section{RESULTS}

This study compared the changes in the condyle of patients before and after orthodontic treatment. 50 condyles of 25 patients (right and left side) ranging from 11 - 40 years of age were evaluated pre and post orthodontic treatment. Condylar changes after orthodontic treatment showed slightly higher female predilection (60\% - females; $40 \%$ - males). The most commonly observed age group to exhibit difference in condylar morphology was 21 - 30 years (48\%) followed by 11 - 20 years ( $40 \%$ ) and the least was found to be in elderly patients of age 31 - 40 years (12\%) demonstrated in Figure 1.

The right and left side condyles of the patients after treatment when assessed, showed that phenotype I was the most commonly observed type on both the sides (44\% right; $44 \%$ - left) in figure 2 and 3 . Figure 4 also demonstrates that the left side condyles of our study population exhibited more changes when compared to the right.

The mean and standard deviation values of the changes in the condyles have been tabulated in Table 1 . The results show that the mean and S.D value on the right side prior to orthodontic treatment was 1.20 and .500 and post treatment was found to be 1.88 and .971 respectively. The mean and S.D value on the left side before treatment was 1.40 and .577 and post treatment was 2.20 and 1.080 respectively. A paired $t$ test was also done to compare the mean and S.D value of the condylar changes pre and post orthodontic treatment on both the sides. The right-side values were - 680 and 0.748 respectively ( $P$ value 0.000 ) and left side values were - 800 and 1.118 respectively which was also statistically significant (P value 0.002 ). 

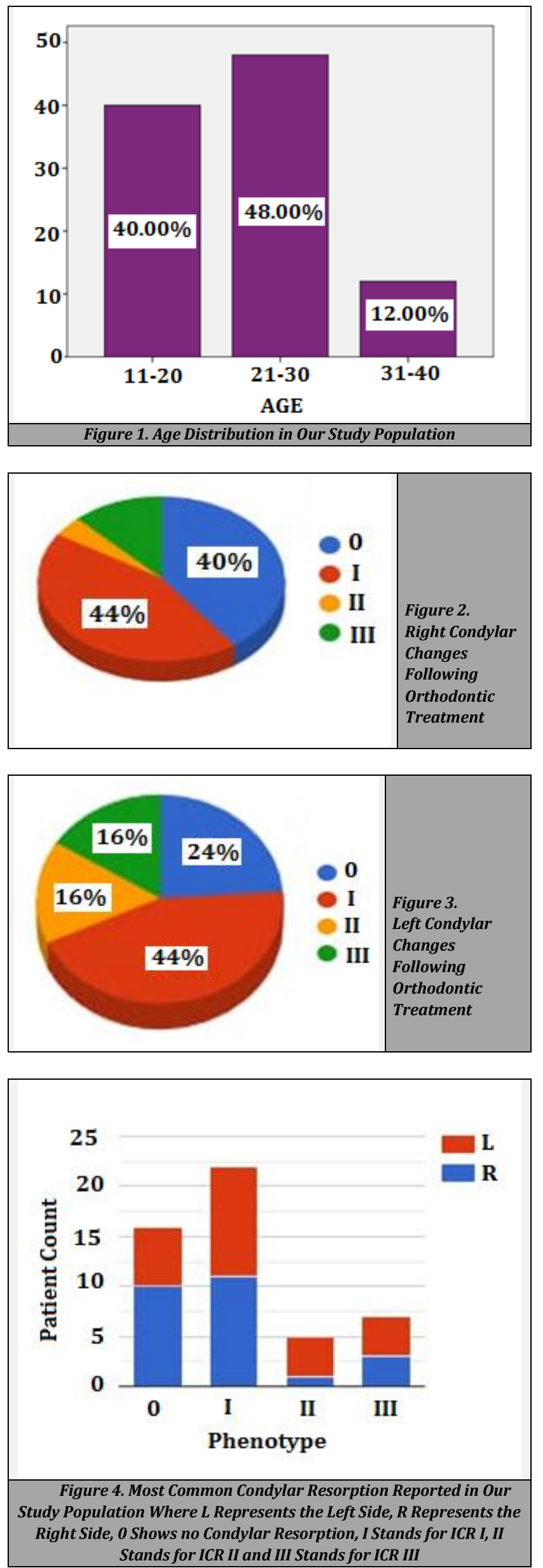

\begin{tabular}{|cccc|}
\hline & Mean & Standard Deviation & P Value \\
\hline Pre-treatment right & 1.20 & .500 & - \\
Pre-treatment left & 1.40 & .577 & - \\
Post treatment right & 1.88 & .971 & - \\
Post treatment left & 2.20 & 1.080 & - \\
Pre and post treatment right & -680 & 0.748 & 0.000 \\
Pre and post treatment left & -800 & 1.118 & 0.002 \\
\hline Table 1 Mean ICR Score Assessed at Pre and Post Orthodontic \\
\multicolumn{3}{|c|}{ Treatment } \\
\hline
\end{tabular}

\section{DISCUSSION}

Idiopathic condylar resorption of the temporomandibular joint is the aggressive degeneration of the condyles with a loss in height which affects the patient both functionally and aesthetically. ${ }^{17}$ A retruded mandibular with clockwise rotation (high angle), malocclusion, Angle Class II anterior open bite (AOB) with excess overjet, loss of overall posterior facial height, TMJ pain are the predominant clinical signs of ICR. ${ }^{18}$ The multifactorial aetiology poses a challenge in diagnosing and managing this pathology. Studies have suggested that orthodontic treatment procedures, which causes an alteration of the mechanical loading of the joint, has an effect on the resorption of the condyles, causing difficulties for patients after treatment.13 Additionally, ICR may also occur following any restorative procedures or orthognathic surgery. For a long time, condylar resorption has been well documented in literature, ${ }^{19}$ but idiopathic condylar resorption has only been described and identified recently due to limited awareness of the condition. Even with a greater recognition, the diagnosis of ICR remains challenging. Radiographic evaluation using panoramic radiographs, lateral cephalometric radiographs, CT scan and MRI serves as an important tool in diagnosis. The affected condyles appear thin and shortened with a flat superior and anterior curvature, basically exhibiting loss of mass. ${ }^{20}$ In the early stages of the disease, when there is slow progression and subtle radiographic changes, patients tend to be asymptomatic making diagnosis difficult. Therefore, idiopathic condylar resorption may go undetected and undiagnosed during routine pre-treatment evaluation of orthodontic patients. ${ }^{21}$ Hence, patients prior to and after orthodontic treatment should be evaluated well to diagnose any clinical occurrences of ICR, if any. This study is done to assess condylar changes before and after orthodontic treatment.

According to the results obtained in this study, females showed a slightly higher occurrence of ICR when compared to male patients. This was in accordance with the study done by Yun - Kyung Hur et al. in Kyungpook National university which involved the lateral cephalometric assessment of 34 patients with condylar resorption. The study also suggested that people of the second and third decades were affected more when compared to other age groups, which was also in concordance. $^{22}$ The female preponderance has been suggested to be in association with the effect of oestrogen and prolactin on bone modulation. ${ }^{6}$ In a study done by Abubaker et al. on the human TMJ, it was found that women with symptoms of TMJ internal derangement had five times more oestrogen and progesterone receptors in the disc when compared to asymptomatic women. ${ }^{23}$

In this study, type I represented normal condylar morphology; type II represented mild cases where the 
transverse diameter was not affected by resorption; type III represented moderate resorption where the transverse diameter was transgressed; type IV represented severe resorption where the head of the condyle exhibited alterations. Type II was the most commonly reported type of condylar change in this study population following orthodontic treatment.

From this study, it is assessed that there are definitive condylar changes following orthodontic treatment and orthodontics is also seen as a criterion causing condylar resorption. This is in accordance with the study done by Kato and his colleagues in which a 12-year-old girl after two years of completion of active orthodontic treatment developed TMJ symptoms at age of 17 . By the age of 21 , the patient was seen with an anterior open bite and long, slender facial appearance. Cephalometric analysis revealed that the ramus was shortened and the condyles were severely deformed and resorbed. ${ }^{24}$ In our study, a paired t test was used to compare the condylar changes bilaterally pre and post orthodontic treatment which revealed significant condylar resorption following orthodontic treatment. A study done by Peltola et al. involving 625 patients, suggested that $9 \%$ condylar flattening occurred after orthodontic treatment. ${ }^{25}$ In 2018, a study done in Saveetha Dental college by Ezhil et al. also suggested that condylar changes occurred after orthodontic treatment. ${ }^{26}$ However, there are studies that have a contrasting opinion stating that orthodontic treatment has no effect on condylar position. Gianelly et al. performed a study using corrected tomograms which evaluated condylar position prior to orthodontic treatment in 37 patients of age 10 - 18 years and compared them with tomograms from 30 treated patients with fixed appliances and the removal of four premolars. No differences in condylar position were noted between the groups. ${ }^{27}$

\section{CONCLUSIONS}

ICR is the result of many predisposing and contributory factors and an accurate and timely diagnosis of this pathology is essential in dental practice. There is a constant need to educate clinicians and help them follow proper guidelines for diagnosis and protocols for treatment of the disorder. The available literature confirms the presence of condylar changes after orthodontic therapy, however more evidencebased high-quality clinical trials with detailed design and long-term follow-up periods need to be conducted yet in order to gain more insight and knowledge on the onset and progression of this pathology.

Data sharing statement provided by the authors is available with the full text of this article at jemds.com.

Financial or other competing interests: None.

Disclosure forms provided by the authors are available with the full text of this article at jemds.com.

The authors are grateful to Saveetha Institute of Medical and Technical Sciences for granting permission to conduct the present study and the faculties of Department of Oral Medicine for their critical review, support during the development and writing of these manuscripts.

\section{REFERENCES}

[1] Young A. Idiopathic condylar resorption: the current understanding in diagnosis and treatment. J Indian Prosthodont Soc 2017;17(2):128-35.

[2] Mercuri LG, Handelman CS. Idiopathic condylar resorption: what should we do? Oral Maxillofac Surg Clin North Am 2020;32(1):105-16.

[3] Wolford LM. Idiopathic condylar resorption of the temporomandibular joint in teenage girls (cheerleaders syndrome). Proc 2001;14(3):246-52.

[4] Papadaki ME, Tayebaty F, Kaban LB, et al. Condylar resorption. Oral Maxillofac Surg Clin North Am 2007;19(2):223-34, vii.

[5] Mercuri LG. Osteoarthritis, osteoarthrosis and idiopathic condylar resorption. Oral Maxillofac Surg Clin North Am 2008;20(2):169-83, v - vi.

[6] Arnett GW, Milam SB, Gottesman L. Progressive mandibular retrusion-idiopathic condylar resorption. Part I. Am J Orthod Dentofacial Orthop 1996;110(1):815.

[7] Arnett GW, Milam SB, Gottesman L. Progressive mandibular retrusion-idiopathic condylar resorption. Part II. American Journal of Orthodontics and Dentofacial Orthopedics 1996;110(2):117-27.

[8] Chouinard AF, Kaban LB, Peacock ZS. Acquired abnormalities of the temporomandibular joint. Oral Maxillofac Surg Clin North Am 2018;30(1):83-96.

[9] Mitsimponas K, Mehmet S, Kennedy R, et al. Idiopathic condylar resorption. $\mathrm{Br} \mathrm{J}$ Oral Maxillofac Surg 2018;56(4):249-55.

[10] Hoppenreijs TJM, Freihofer HPM, Stoelinga PJW, et al. Condylar remodelling and resorption after Le Fort I and bimaxillary osteotomies in patients with anterior open bite. International Journal of Oral and Maxillofacial Surgery 1998;27(2):81-91.

[11] Krajenbrink TGA. The silhouette of the mandibular condyle on radiographs. Rijksuniversiteit te Groningen 1994.

[12] Gomes LR, Gomes M, Jung B, et al. Diagnostic index of 3D osteoarthritic changes in TMJ condylar morphology. Proc SPIE Int Soc Opt Eng 2015; 9414:941405.

[13] De Clercq CA, Neyt LF, Mommaerts MY, et al. Condylar resorption in orthognathic surgery: a retrospective study. Int J Adult Orthodon Orthognath Surg 1994;9(3):233-40.

[14] Arnett GW, Tamborello JA. Progressive class II development: female idiopathic condylar resorption. Oral Maxillofac Surg Clin North Am 1990;2(4):699-716.

[15] Crawford JG, Stoelinga PJ, Blijdorp PA, et al. Stability after reoperation for progressive condylar resorption after orthognathic surgery: report of seven cases. J Oral Maxillofac Surg 1994;52(5):460-6.

[16] He Y, Lin H, Lin Q, et al. Morphologic changes in idiopathic condylar resorption with different degrees of bone loss. Oral Surg Oral Med Oral Pathol Oral Radiol 2019;128(3):332-40.

[17] Huang YL, Pogrel MA, Kaban LB. Diagnosis and management of condylar resorption. J Oral Maxillofac Surg 1997;55(2):114-9; discussion 119-20.

[18] Handelman CS. Ask us. Condylar resorption. Am J Orthod Dentofacial Orthop 2004;125(2):16A. 
[19] Burke PH. A case of acquired unilateral mandibular condylar hypoplasia. Proc R Soc Med 1961;54(6):507-10.

[20] Hoppenreijs TJ, Stoelinga PJ, Grace KL, et al. Long- term evaluation of patients with progressive condylar resorption following orthognathic surgery. International Journal of Oral and Maxillofacial Surgery 1999;28(6):411-8.

[21] Stappert D, Warburton G. Recognition of idiopathic condylar resorption as a diagnostic possibility in an academic orthodontic clinic. J Orthod Endo 2015;1(2):14.

[22] Hur YK, Park HS, Choi JK. Lateral cephalometic assessment in patients with condylar resorption. Journal of Oral Medicine and Pain 2006;31(4):337-46.

[23] Abubaker AO, Raslan WF, Sotereanos GC. Estrogen and progesterone receptors in temporomandibular joint discs of symptomatic and asymptomatic persons: a preliminary study. Journal of Oral and Maxillofacial
Surgery 1993;51(10):1096-100.

[24] Kato Y, Hiyama S, Kuroda T, et al. Condylar resorption 2 years following active orthodontic treatment: a case report. Int J Adult Orthodon Orthognath Surg 1999;14(3):243-50.

[25] Peltola JS, Nyström M, Könönen M, et al. Radiographic structural findings in the mandibular condyles of young individuals receiving orthodontic treatment. Acta Odontol Scand 1995;53(2):85-91.

[26] Ezhil I, Arun AV, Kumar MPS. Morphological changes of the mandibular condyle following orthodontic treatment. Drug Invention Today 2018;10(Spec Iss 1):2802-04.

[27] Gianelly AA, Hughes HM, Wohlgemuth P, et al. Condylar position and extraction treatment. Am J Orthod Dentofacial Orthop 1988;93(3):201-5. 